\section{BMJ Paediatrics Open}

\title{
Copper and zinc concentrations in the breast milk of mothers undergoing treatment for Wilson's disease: a prospective study
}

\author{
Hiroko Kodama (D) , ${ }^{1,2}$ Yasumi Anan, ${ }^{3}$ Yoichi Izumi, ${ }^{2}$ Yasuhiro Sato, ${ }^{2}$ \\ Yasumitsu Ogra (D) ${ }^{4}$
}

To cite: Kodama H, Anan Y, Izumi Y, et al. Copper and zinc concentrations in the breast milk of mothers undergoing treatment for Wilson's disease: a prospective study. BMJ Paediatrics Open 2021;5:e000948. doi:10.1136/ bmjpo-2020-000948

- Additional supplemental material is published online only. To view, please visit the journal online (http://dx.doi.org/ 10.1136/bmjpo-2020-000948).

Received 4 January 2021 Accepted 1 June 2021

Check for updates

(C) Author(s) (or their employer(s)) 2021. Re-use permitted under CC BY-NC. No commercial re-use. See rights and permissions. Published by BMJ.

${ }^{1}$ Graduate School of Health Sciences, Master's prgram in Health and Dietetics, Teikyo Heisei University - Ikebukuro Campus, Toshima-ku, Japan ${ }^{2}$ Department of Pediatrics, Teikyo University School of Medicine Graduate School of Medicine, Itabashi-ku, Japan ${ }^{3}$ Faculty of Environmental \& Symbiotic Sciences, Prefectural University of Kumamoto, Kumamoto, Japan

${ }^{4}$ Graduate School of

Pharmaceutical Sciences, Chiba University, Chiba, Japan

Correspondence to Dr Hiroko Kodama; kodamah2018@gmail.com

\section{ABSTRACT}

Objective To evaluate the concentrations of copper and zinc in the breast milk of mothers undergoing treatment for Wilson's disease (WD) and clarify whether they can safely breast feed their infants.

Design This was an observational and prospective study in an individual-based case series.

Setting Breast milk samples were collected from participants across Japan from 2007 to 2018 at the Department of Pediatrics, Teikyo University in Tokyo. This was a primary-care level study. Clinical data were collected from the participants' physicians.

Patients Eighteen Japanese mothers with WD who were treated with trientine, penicillamine or zinc, and 25 healthy mothers as controls, were enrolled.

Main outcome measures Whey exacted from the milk was used to evaluate the distribution of copper by high-performance liquid chromatography-inductively coupled plasma mass spectrometry. Copper and zinc concentrations in the breast milk samples were analysed by atomic absorption spectrometry.

Results Copper distribution was normal in the breast milk of mothers with WD treated with trientine, penicillamine or zinc. No peak was detected for trientinebound or penicillamine-bound copper. The mean copper concentrations in the mature breast milk of patients treated with trientine, penicillamine and zinc were 29.6, 26 and $38 \mu \mathrm{g} / \mathrm{dL}$, respectively, and were within the normal range compared with the value in healthy controls $(33 \mu \mathrm{g} /$ $\mathrm{dL})$. Likewise, mean zinc concentrations were normal in the mature breast milk of patients treated with trientine and penicillamine (153 and $134 \mu \mathrm{g} / \mathrm{dL}$, respectively vs $160 \mu \mathrm{g} / \mathrm{dL}$ in healthy controls). Zinc concentrations in the breast milk of mothers treated with zinc were significantly higher than those in control milk. All infants were born normally, breast fed by mothers undergoing treatment and exhibited normal development.

Conclusions Our results suggest that mothers with WD can safely breast feed their infants, even if they are receiving treatment for WD.

\section{INTRODUCTION}

Wilson's disease (WD) is an autosomal recessive copper toxicosis caused by a mutation in $A T P 7 B$ and shows a prevalence of
What is known about the subject

Patients with Wilson's disease (WD) are required to adhere to therapeutic regimens throughout their life, including during pregnancy and after delivery. However, clinical practice guidelines for WD indicate that breast feeding is not recommended in mothers with WD under drug therapy. Meanwhile, the safety of breast feeding by mothers undergoing treatment for WD has been evaluated by a limited number of studies.

\section{What this study adds}

This study revealed normal copper and zinc concentrations in breast milk from mothers undergoing treatment for WD. Moreover, no trientine-copper or penicillamine-copper was detected in breast milk from mothers with WD treated with trientine or penicillamine, respectively. The infants were born normally, breast fed by mothers undergoing treatment during lactation, and exhibited normal development. These results indicate that mothers undergoing treatment for WD can safely breast feed their infants.

approximately 1 per 30000 people. ${ }^{1-4}$ ATP7B transports copper from the cytosol to the Golgi apparatus in normal hepatocytes, from which it is excreted from hepatocytes into the blood as ceruloplasmin-bound copper. Ceruloplasmincopper accounts for approximately 90\%-95\% of the serum copper levels. ${ }^{13}$ Linder reported that apoceruloplasmin concentrations in blood plasma are substantial, and there are numerous other copper-binding components in the blood beyond ceruloplasmin (which may account for only $50 \%-70 \%$ of the total copper). ${ }^{5}$ In patients with WD, copper cannot be transported from the cytosol to the Golgi apparatus. As a result, copper accumulates in the hepatocytes, and the serum levels of copper and ceruloplasmin decrease. Copper 
can also be released from damaged hepatocytes into the blood, accumulating in extrahepatic tissues including the brain, kidney and cornea. ${ }^{14}$ The prominent features of WD are hepatic and neurological diseases. Standard treatment involves administration of a chelator (penicillamine or trientine) or zinc throughout the patient's lifetime. Chelators enhance the excretion of copper into the urine, whereas oral administration of zinc inhibits copper absorption in the intestine, resulting in a negative copper balance. If good control is achieved through treatment, patients with WD can live normal lives; female patients can even become pregnant, although treatment should be continued during pregnancy. ${ }^{1-5}$

The product instructions for penicillamine note that "nursing should be discontinued during treatment with this drug, because it is unknown whether penicillamine can pass into the breast milk'. ${ }^{6}$ This is also the case for zinc acetate. ${ }^{7}$ Meanwhile, product instructions for trientine do not mention lactation. ${ }^{8}$ Moreover, practice guidelines for WD from the European Association for the Study of the Liver ${ }^{2}$ and American Association for the Study of Liver Diseases ${ }^{3}$ describe that breast feeding under drug therapies is not recommended for mothers with WD. Notably, recent review articles in the field have also mentioned that breast feeding is not recommended for mothers with WD. ${ }^{45}$

Breast feeding is, however, recommended worldwide for the health of the infant and mother. ${ }^{9-12}$ Meanwhile, in mothers with WD, who should continue drug treatment for WD during lactation, the effects of drugs on lactation are unclear. In this study, we examined the safety of breast feeding by mothers with WD.

\section{METHODS}

\section{Subjects and biological samples}

Eighteen Japanese mothers with WD were enrolled in this study. Four and seven of these mothers were enrolled to collect the colostrum and transitional milk, respectively. These mothers were also enrolled to collect mature milk samples. The remaining seven mothers were enrolled to collect mature milk (see online supplemental table S1). The colostrum and transitional milk samples were obtained at 0-4 and 5-13 days after delivery, respectively, according to Ballard and Morrow. ${ }^{13}$ In contrast, the time after delivery at which mature milk samples were collected differed for each mother, as shown in online supplemental table S1, with a mean of 96 (14-337) days after delivery. The patients were diagnosed with WD based on their liver copper concentrations, $A T P 7 B$ analysis and/ or biochemical data and had been continuously treated with trientine, penicillamine or zinc since their initial diagnosis. The mean ages at diagnosis and pregnancy were 13.9 years (7-29) and 31.8 years (23-38), respectively. The numbers of patients with WD with hepatic or neurological manifestations were 14 and 4, respectively. The numbers of patients treated with trientine, penicillamine, zinc or a combination of zinc and trientine were
4, 7, 6 and 1, respectively. All infants were born without complications and breast fed by mothers undergoing treatment during lactation. When the mature breast milk samples were collected, the mothers stated that the infants showed normal development.

Patients' sera were collected during pregnancy. Control breast milk samples were obtained from 25 healthy mothers with a mean age of 32.1 years (26-40 years). The breast milk samples were frozen until use.

\section{Determination of copper distribution profiles in breast milk}

The breast milk copper distribution profiles were assessed as described previously. ${ }^{14}$ Briefly, the frozen mature milk samples were thawed and centrifuged at 105 $000 \times \mathrm{g}$ for $60 \mathrm{~min}$ at $4^{\circ} \mathrm{C}$ and the resulting supernatant (whey fraction) was used. A $20 \mu \mathrm{L}$ aliquot of the copperpenicillamine complex solution $(1.14 \mathrm{mM})$, coppertrientine complex solution $(1.67 \mathrm{mM})$ or whey was used for high-performance liquid chromatography-inductively coupled plasma mass spectrometry (HPLC-ICP-MS) to analyse the distribution of copper, as described previously. ${ }^{14}$ The HPLC system (Prominence, Shimadzu, Kyoto, Japan) consisted of an online degasser, an HPLC pump, a Rheodyne six-port injector and a multi-mode size exclusion column (Shodex GS-520HQ, exclusion size $>300000 \mathrm{kDa}, 7.5$ i.d. $\times 300 \mathrm{~mm}$ with a guard column; Showa Denko, Tokyo, Japan). The sample was eluted with $50 \mathrm{mM}$ Tris- $\mathrm{HCl}, \mathrm{pH} 7.4$, at a flow rate of $0.6 \mathrm{~mL} /$ $\mathrm{min}$. The eluate was introduced directly into the ICP-MS (Agilent 7500, Agilent Technologies, Santa Clara, California, USA) to detect $\mathrm{Cu}$ at $m / z 65$.

\section{Determination of copper and zinc concentrations in breast milk}

Frozen milk samples were thawed and diluted with ultrapure ion-exchange water. Copper and zinc concentrations in the diluted breast milk samples were analysed by atomic absorption spectrometry (Hitachi Z6100, Tokyo, Japan) at wavelengths of $324.8 \mathrm{~nm}$ for copper and $213.9 \mathrm{~nm}$ for zinc, as reported previously. ${ }^{15}$

\section{Statistical analysis}

The results are expressed as the means and SD or as medians (online supplemental table S1). For statistical analysis, at least five samples of mature milk were collected from each group. Statistical comparisons between control mature milk samples and mature milk samples of mothers treated with each drug were performed using the MannWhitney $\mathrm{U}$ test. $\mathrm{P}$ values $<0.05$ were considered statistically significant. Statistical analysis was not performed for colostrum or transitional milk samples due to the small sample size (1-3 samples per group).

\section{Patient and public involvement}

Patients or the public were not involved in the design, conduct, reporting or dissemination plans of our research. 


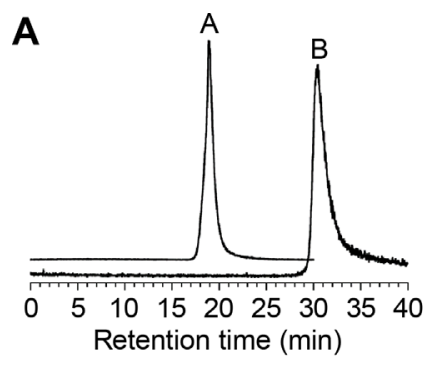

B
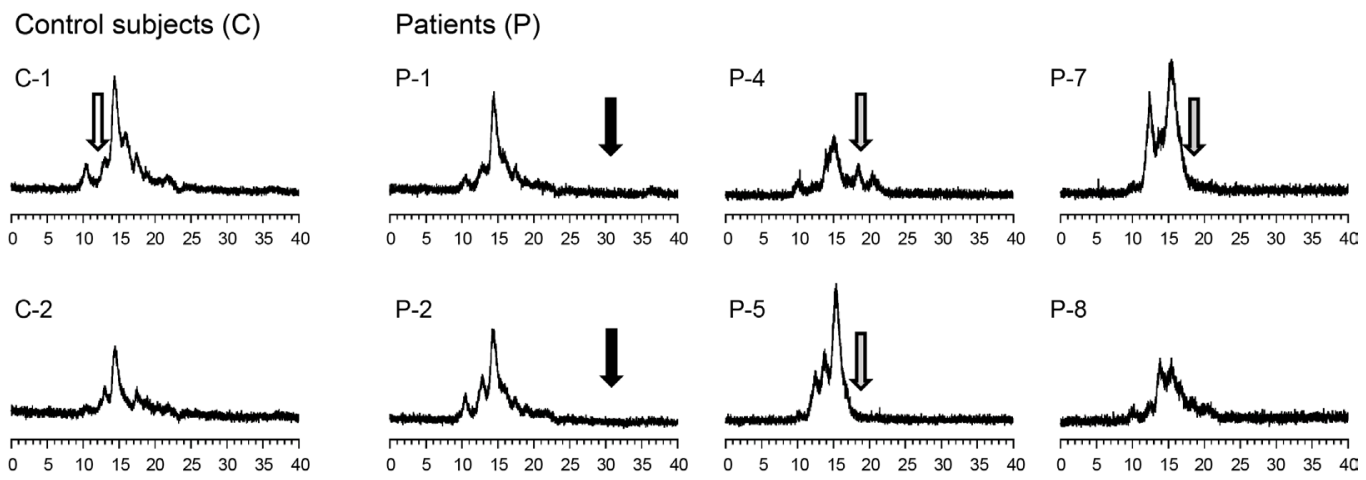

$\mathrm{P}-8$
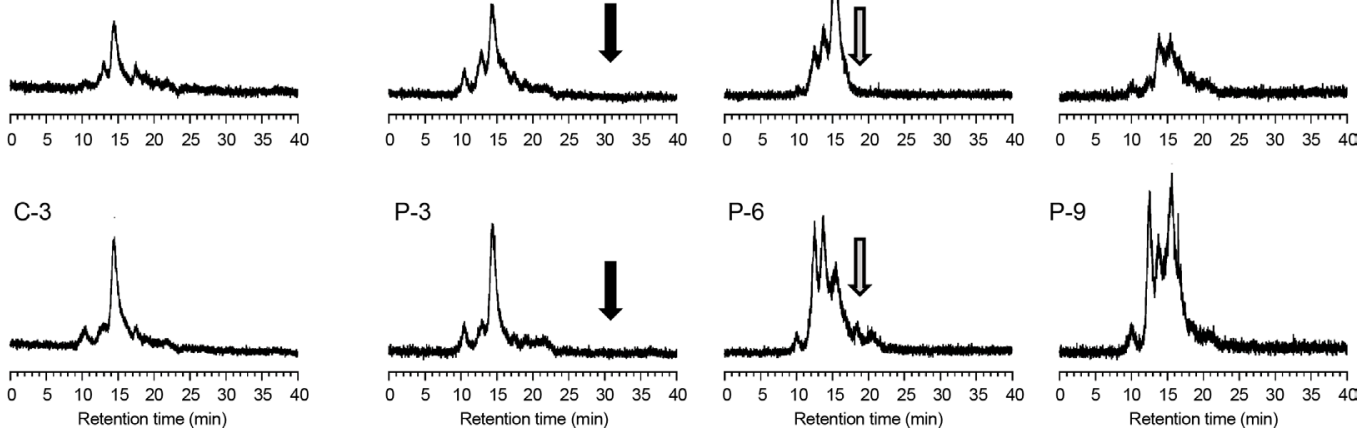

Figure 1 Elution profiles of copper obtained by high-performance liquid chromatography-inductively coupled plasma mass spectrometry. (A) Standard compounds, that is, copper-penicillamine (A) and copper-trientine (B) complexes were eluted at retention times of 19 and $30.5 \mathrm{~min}$, respectively. (B) Copper distribution in mature milk samples collected from control subjects and mothers treated with trientine, penicillamine or zinc. White, grey and black arrows indicate the retention times of ceruloplasmin, a copper-penicillamine complex and a copper-trientine complex, respectively. Lactalbumin-bound copper showed the highest peak in the elution profiles. The ceruloplasmin-bound copper peak was not observed in mature breast milk from the controls or patients.

\section{RESULTS}

The HPLC-ICP-MS peaks corresponding to the penicillamine-copper and trientine-copper complexes were eluted at retention times of 19 and $30.5 \mathrm{~min}$, respectively (figure 1A) and were not detected in the breast milk of mothers treated with these drugs (figure 1B). The distribution profiles of breast milk were similar between the mothers with WD and control mothers; all milk samples showed a pattern similar to that of mature breast milk from healthy mothers, as reported previously. ${ }^{14}$ The highest peak in HPLC-ICP-MS appeared to be that of lactalbumin-bound copper, based on comparison of the retention time with that described previously. ${ }^{14}$

Tables 1 and 2 show the copper and zinc concentrations in the breast milk. The zinc concentration in control milk was significantly high in the colostrum, after which it decreased to concentrations similar to those reported previously. ${ }^{1516}$

As shown in tables 1 and 2, the serum copper concentrations were low in mothers with WD. The copper concentration in the colostrum from two mothers treated with trientine was slightly low in one subject and slightly high in the other compared with that in the colostrum from controls. The copper concentration in the colostrum from mothers treated with penicillamine or zinc was also slightly high, however, the difference compared with controls was not significant.

The zinc concentration in the colostrum from a mother treated with trientine was significantly high $(1446 \mu \mathrm{g} /$ $\mathrm{dL}$ ); the reason for this result is unknown. However, zinc concentrations decreased to the normal range in transitional and mature milk in the same mother (Patient No. 3 in online supplemental table S1). In the colostrum from another mother treated with trientine, the zinc concentration was normal. The copper concentration in transitional milk was slightly lower in a mother treated with trientine and a mother treated with zinc compared with the minimum levels in the control samples.

In contrast, the copper and zinc concentrations were normal in mature breast milk from mothers treated with trientine and penicillamine, despite the low copper concentrations in the serum of the mothers. The zinc concentration in the breast milk of patients treated with zinc was significantly higher than the zinc concentration 


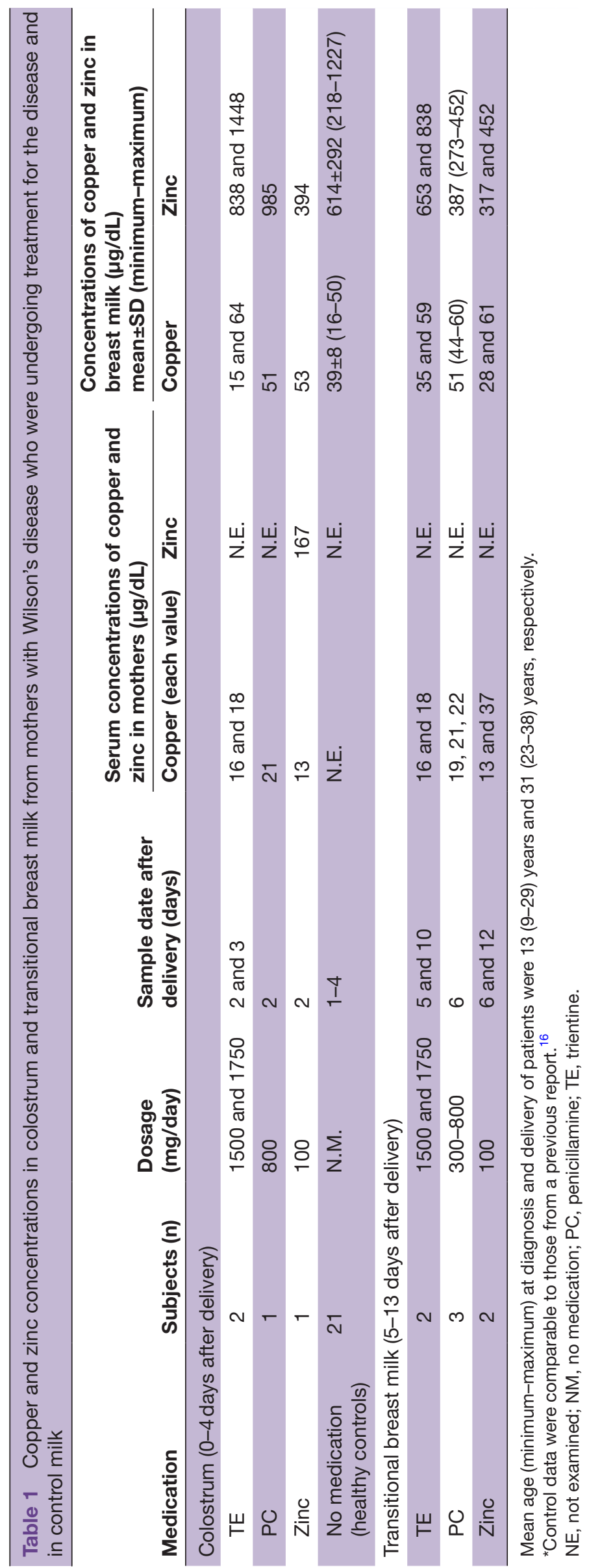

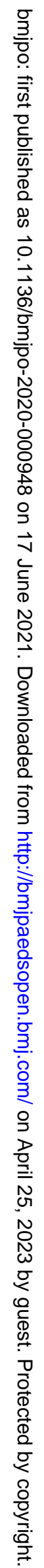



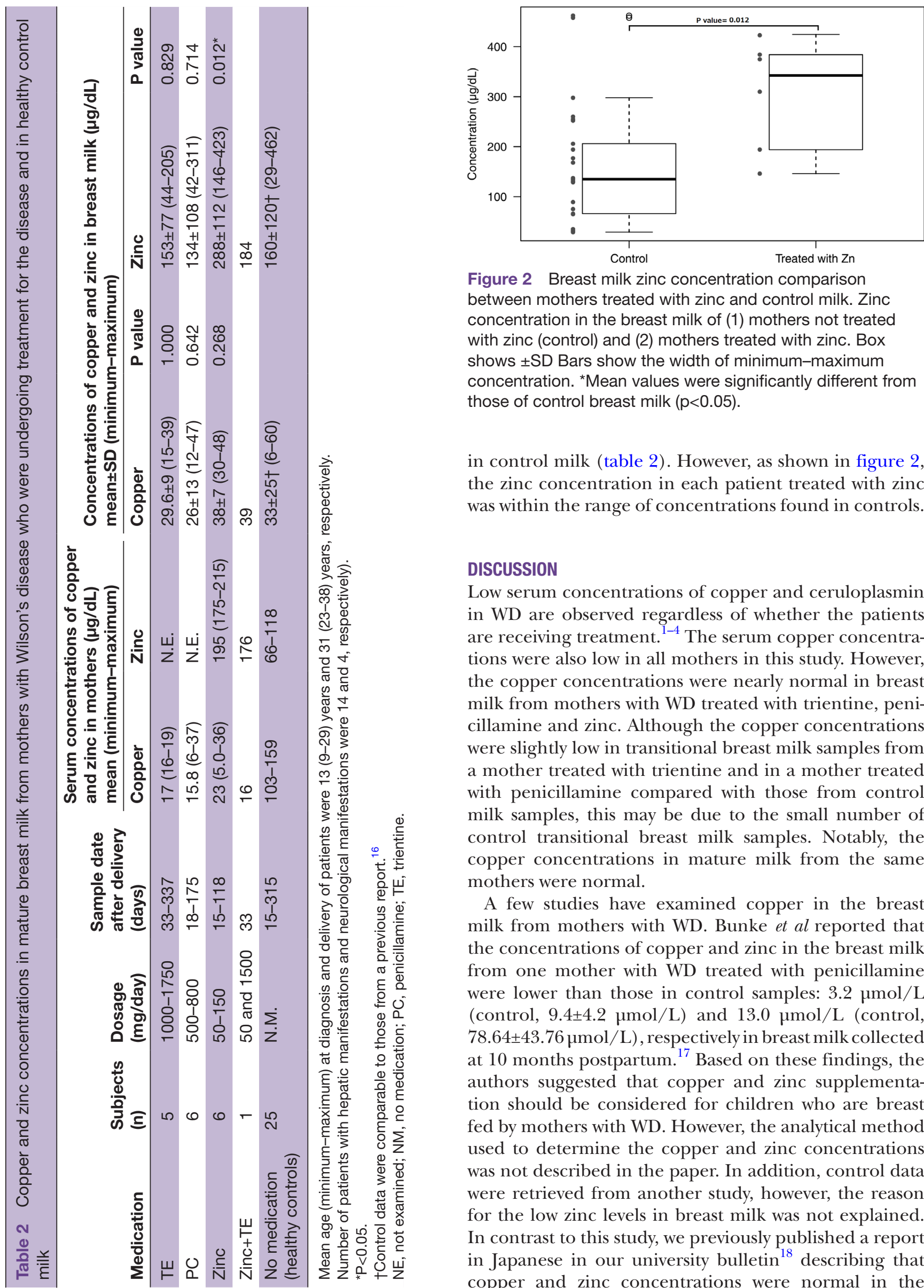

Figure 2 Breast milk zinc concentration comparison between mothers treated with zinc and control milk. Zinc concentration in the breast milk of (1) mothers not treated with zinc (control) and (2) mothers treated with zinc. Box shows \pm SD Bars show the width of minimum-maximum concentration. *Mean values were significantly different from those of control breast milk $(p<0.05)$.

in control milk (table 2). However, as shown in figure 2, the zinc concentration in each patient treated with zinc was within the range of concentrations found in controls.

\section{DISCUSSION}

Low serum concentrations of copper and ceruloplasmin in WD are observed regardless of whether the patients are receiving treatment. ${ }^{1-4}$ The serum copper concentrations were also low in all mothers in this study. However, the copper concentrations were nearly normal in breast milk from mothers with WD treated with trientine, penicillamine and zinc. Although the copper concentrations were slightly low in transitional breast milk samples from a mother treated with trientine and in a mother treated with penicillamine compared with those from control milk samples, this may be due to the small number of control transitional breast milk samples. Notably, the copper concentrations in mature milk from the same mothers were normal.

A few studies have examined copper in the breast milk from mothers with WD. Bunke et al reported that the concentrations of copper and zinc in the breast milk from one mother with WD treated with penicillamine were lower than those in control samples: $3.2 \mu \mathrm{mol} / \mathrm{L}$ (control, 9.4 $4.2 \mu \mathrm{mol} / \mathrm{L}$ ) and $13.0 \mu \mathrm{mol} / \mathrm{L}$ (control, $78.64 \pm 43.76 \mu \mathrm{mol} / \mathrm{L}$ ), respectively in breast milk collected at 10 months postpartum. ${ }^{17}$ Based on these findings, the authors suggested that copper and zinc supplementation should be considered for children who are breast fed by mothers with WD. However, the analytical method used to determine the copper and zinc concentrations was not described in the paper. In addition, control data were retrieved from another study, however, the reason for the low zinc levels in breast milk was not explained. In contrast to this study, we previously published a report in Japanese in our university bulletin ${ }^{18}$ describing that copper and zinc concentrations were normal in the 
breast milk from nine mothers with WD who were treated with trientine, penicillamine or zinc. Isagawa et $a l^{19}$ also reported that the zinc concentration in breast milk from a mother treated with zinc was normal compared with control breast milk.

HPLC-ICP-MS analysis of mature breast milk from control subjects and mothers with WD showed no peak for ceruloplasmin. Suzuki et al and Kiyosawa et al observed similar results in mature breast milk from healthy mothers, although ceruloplasmin was detected in the colostrum. ${ }^{1420}$ These findings, along with our results, indicate that ceruloplasmin is rarely involved in the secretion of copper into mature breast milk. Although the serum ceruloplasmin concentrations in mothers with WD were significantly lower than those in healthy control mothers, the copper concentration in breast milk from mothers with WD and control samples was similar, supporting that ceruloplasmin is not involved in copper secretion into milk.

The results of HPLC-ICP-MS also showed no peak for copper sequestered by trientine or penicillamine in breast milk from mothers with WD treated with these drugs. Using this analysis method, copper-unbound chelators cannot be detected. In addition, the concentrations of trientine and penicillamine were not examined in this study. However, it has been reported that free trientine was not detected in the serum of rats administered trientine. ${ }^{21}$ Another study demonstrated that in rats administered penicillamine, nearly all penicillamine in the plasma was bound to plasma proteins. ${ }^{22}$ These results suggest that chelator drugs are rarely transferred to the milk.

In addition, babies breast fed by mothers with WD undergoing treatment exhibited normal development in our study. Our results clearly showed that the copper and zinc concentrations in breast milk from WD mothers treated with trientine, penicillamine or zinc were normal, and that trientine and penicillamine (copper-bound) were not transported into the breast milk nor did they affect the infants.

Long-Evans Cinnamon (LEC) rats, as well as toxic milk (tx) and $a t p 7 b$ knockout mice are commonly used as WD animal models. ${ }^{23}$ Although LEC rats have been shown to have low serum copper concentrations, the corresponding copper concentrations in breast milk have not been examined. However, the pups develop normally when fed breast milk from female LEC rats, suggesting that the breast milk is not abnormal. In contrast, tx mice produce copper-deficient breast milk causing the pups to die from copper deficiency. There are two types of tx mice; the first has a naturally occurring point mutation in $a t p 7 b$ and the second, toxic milk Jackson (txj) mice, arose spontaneously and harbour a single-nucleotide mutation in $a t p 7 b$. Both types of tx mice produce copper-deficient milk. In $a t p 7 b$ knockout mice, copper levels in the milk of mutant females are also low, as observed in tx mice. These findings suggest that there are apparent speciesspecific differences in copper excretion from breast cells into breast milk, at least considering humans (WD), rats (LEC) and mice (tx, txj, atp $7 b-\mathrm{KO})$.

ATP7A and ATP7B are copper-transporting ATPases with similar structures and functions, ${ }^{124}$ both of which are localised on the membrane of the Golgi apparatus and transfer copper from the cytosol into the Golgi apparatus for subsequent copper excretion. ATP7A is found in intestinal cells, transporting copper from enterocytes into the blood. ${ }^{1}$ Mutations in ATP7A are associated with Menkes disease, which involves symptoms of severe copper deficiency. ${ }^{1}$ In contrast, ATP7B transfers copper from the cytosol to the trans-Golgi network of hepatocytes. Subsequently, copper in the trans-Golgi network is incorporated into apo-ceruloplasmin and secreted as ceruloplasmin into the blood. ATP7B also excretes copper into the bile.

Both $A T P 7 A$ and $A T P 7 B$ are expressed in various organs, including the kidneys, brain, lungs, placenta and mammary glands. ${ }^{1}$ However, the function of ATP7B in these organs remains unclear. It has been reported that ATP7B is also expressed in human breast adenocarcinoma cells, although its function is unclear. ${ }^{25}$ Meanwhile, in human breast cells, ATP7A may be involved in the cellular efflux of copper into milk. ${ }^{26}$ ATP7A was also detected in both luminal and serosal membranes, which suggests a dual role, that is, exporting copper into milk and importing it back into maternal circulation. ${ }^{24}{ }^{27} \mathrm{In}$ contrast, as $a t p 7 b$ is the major copper transporter in the mammary glands of mice, tx mice and atp $7 b-\mathrm{KO}$ mice show impaired copper transport into milk, causing the copper concentration in milk to be significantly lower than that in control mice. ${ }^{28}$ Hence, further studies are needed to clarify the species-specific differences in copper excretion from breast cells into milk.

Hence, our study proposes that since treatment with penicillamine, trientine or zinc maintains the normal concentrations of copper and zinc in the breast milk of breastfeeding patients with $\mathrm{WD}$, these agents should be the preferred therapeutic choice for such patients. HPLC-ICP-MS analysis further revealed that the copper distribution profiles in mature breast milk from mothers with WD undergoing drug therapy were normal compared with those in milk from control mothers. In addition, no peak for trientine-copper or penicillaminecopper was observed in milk from mothers treated with trientine and penicillamine, respectively. Further studies of zinc concentrations in breast milk of mothers on zinc therapy are needed in view of our findings. All infants were born normally, breast fed by mothers undergoing treatment during lactation and exhibited normal development. These results suggest that mothers undergoing treatment for WD can safely breast feed their infants.

Acknowledgements We would like to thank Editage (www.editage.com) for English language editing.

Contributors HK conceived this study. HK, YI and YS obtained informed consent from the participants and collected the breast milk samples. YO and YA conducted HPLC-ICP-MS analysis and measured copper and zinc concentrations in the breast 
milk samples. YO generated the figures. HK wrote the first draft of the manuscript. All authors approved the final version of the manuscript.

Funding This work was supported by MHLW Research on Rare and Intractable Diseases, Programme Grant Number JPMH20FC1025, from the Ministry of Health, Labour and Welfare in Japan.

Disclaimer The funding body had no role in the study design; in the collection, analysis and interpretation of the data; in the writing of the report; or in the decision to submit the paper for publication.

Competing interests None declared.

Patient consent for publication Obtained.

Ethics approval This study was approved by Teikyo University Ethical Review Board for Medical and Health Research Involving Human Subjects (No. 07-096) and informed consent for this study in writing was obtained from all patients. Consent for publication in BMJ was obtained from 16 out of 18 patients. Furthermore, consent could not be obtained from the remaining two patients, as their mailing addresses had changed, and they could not be contacted.

Provenance and peer review Not commissioned; externally peer reviewed.

Data availability statement Data are available upon reasonable request. Data are available from the corresponding author upon reasonable request.

Supplemental material This content has been supplied by the author(s). It has not been vetted by BMJ Publishing Group Limited (BMJ) and may not have been peer-reviewed. Any opinions or recommendations discussed are solely those of the author(s) and are not endorsed by BMJ. BMJ disclaims all liability and responsibility arising from any reliance placed on the content. Where the content includes any translated material, BMJ does not warrant the accuracy and reliability of the translations (including but not limited to local regulations, clinical guidelines, terminology, drug names and drug dosages), and is not responsible for any error and/or omissions arising from translation and adaptation or otherwise.

Open access This is an open access article distributed in accordance with the Creative Commons Attribution Non Commercial (CC BY-NC 4.0) license, which permits others to distribute, remix, adapt, build upon this work non-commercially, and license their derivative works on different terms, provided the original work is properly cited, appropriate credit is given, any changes made indicated, and the use is non-commercial. See: http://creativecommons.org/licenses/by-nc/4.0/.

ORCID iDs

Hiroko Kodama http://orcid.org/0000-0001-9612-0434

Yasumitsu Ogra http://orcid.org/0000-0002-8268-1828

\section{REFERENCES}

1 Kodama H, Fujisawa C, Bhadhprasit W. Inherited copper transport disorders: biochemical mechanisms, diagnosis, and treatment. Curr Drug Metab 2012;13:237-50.

2 European Association for Study of Liver. EASL clinical practice guidelines: Wilson's disease. J Hepatol 2012;56:671-85.

3 Roberts EA, Schilsky ML, American Association for Study of Liver Diseases (AASLD). Diagnosis and treatment of Wilson disease: an update. Hepatology 2008;47:2089-111.

4 Członkowska A, Litwin T, Dusek P, et al. Wilson disease. Nat Rev Dis Primers 2018:4:21.

5 Linder MC. Apoceruloplasmin: abundance, detection, formation, and metabolism. Biomedicines 2021;9:233.
6 Cuprimine®: penicillamine capsules. full prescribing information. Available: https://cuprimine.com/ [Accessed 24 Sep 2020].

7 Drug Label Information. GALZIN@: Zinc acetate capsule. Available: https://dailymed.nlm.nih.gov/dailymed/druglnfo.cfm?setid= a0c72bff-20f3-4241-b966-34a95178d1a3 [Accessed 24 Sep 2020].

8 Full Prescribing Information. Syprine $₫$ : trientine hydrochloride capsules. Available: https://syprine.com/ [Accessed 24 Sep 2020].

9 Cattaneo A, Burmaz T, Arendt M. Protection, promotion and support of breastfeeding in Europe. Public Health Nur 2009;28:1-9.

10 Section on Breastfeeding. Breastfeeding and the use of human milk. Pediatrics 2012;129:e827-41.

11 Victora CG, Bahl R, Barros AJD, et al. Breastfeeding in the $21^{\text {st }}$ century: epidemiology, mechanisms, and lifelong effect. Lancet 2016;387:475-90.

12 Chowdhury R, Sinha B, Sankar MJ, et al. Breastfeeding and maternal health outcomes: a systematic review and meta-analysis. Acta Paediatr 2015;104:96-113.

13 Ballard O, Morrow AL. Human milk composition: nutrients and bioactive factors. Pediatr Clin North Am 2013;60:49-74.

14 Suzuki KT, Tamagawa H, Hirano S, et al. Changes in element concentration and distribution in breast-milk fractions of a healthy lactating mother. Biol Trace Elem Res 1991;28:109-21.

15 Higashi A, Ikeda T, Uehara I, et al. Zinc and copper contents in breast milk of Japanese women. Tohoku J Exp Med 1982;137:41-7.

16 Yamawaki N, Yamada M, Kan-no T, et al. Macronutrient, mineral and trace element composition of breast milk from Japanese women. J Trace Elem Med Biol 2005;19:171-81.

17 Bunke VH, Cario WR, Schneider M. Abweichyngen in Der zusammemsetzung Der muttermilch bei morbus Wilson. KinderarztI Prax 1989;57:89-92.

18 Izumi Y. Can a mother with Wilson's disease give her breast milk to their infant? Teikyo Med J 2012;35:17-24. (in Japanese).

19 Isagawa S, Shohira H, Hokama N. Measurement of zinc concentration in blood and breast milk of a Wilson's disease patient taking zinc acetate. Pharmazie 2020;75:176-7.

20 Kiyosawa I, Matsuyama J, Nyui S, et al. Ceruloplasmin concentration in human colostrum and mature milk. Biosci Biotechnol Biochem 1995;59:713-4

21 Miyasaki K, Kishino S, Kobyashi M, et al. Determination of triethylenetetramine in plasma of patients by high-performance liquid chromatography. Chem. Pharm. Bull. 1990;38:1035-8.

22 Nozu T, Suwa T, Fukushima K. Metabolic fate of 14C-labeled D-penicillamine (II) metabolism and absorption, distribution and excretion after repeated administration in rats. Ouyou Yakuri 1977:14:277-87. in Japanese, English abstract.

23 Reed E, Lutsenko S, Bandmann O. Animal models of Wilson disease. J Neurochem 2018:146:356-73.

24 Lutsenko S, Barnes NL, Bartee MY, et al. Function and regulation of human copper-transporting ATPases. Physiol Rev 2007;87:1011-46.

25 Michalczyk A, Bastow $\mathrm{E}$, Greenough $\mathrm{M}$, et al. Atp7B expression in human breast epithelial cells is mediated by lactational hormones. $J$ Histochem Cytochem 2008;56:389-99.

26 Ackland ML, Anikijenko P, Michalczyk A, et al. Expression of Menkes copper-transporting ATPase, MNK, in the lactating human breast: possible role in copper transport into milk. $J$ Histochem Cytochem. 1999;47:1553-61.

27 Wadwa J, Chu Y-H, Nguyen N, et al. Effects of ATP7A overexpression in mice on copper transport and metabolism in lactation and gestation. Physiol Rep 2014;2:e00195.

28 Michalczyk AA, RIEGER J, ALLEN KJ, et al. Defective localization of the Wilson disease protein (ATP7B) in the mammary gland of the toxic milk mouse and the effects of copper supplementation. Biochem J 2000;352:565-71. 